\title{
Sticholysin II Identifies Intracellular Lipid Deposits in Niemann Pick C Fibroblasts
}

\begin{abstract}
Keywords: Sticholysin II; Niemann Pick C disease; Sphingomyelin; Cholesterol; Cholera toxin filipin; Confocal microscopy; Human fibroblasts; Lysosomal diseases

Abstract

The cells of patients of Niemann-Pick $C$ disease are characterized by the presence of sphingolipids and cholesterol atypical intracellular storages. The existence of a cause-effect relation between the composition, nature and kinetics of the lipid storages and the pathogenesis of the disease still not clear, mainly due to the scarcity and low reliability of the tools that are able to unambiguously recognize the different lipids. In this study we report the use of sticholysine II, a sea-anemone protein able to bind to sphingomyelin, along with its antibody in the analysis of cell lipids. Results demonstrate that the protein recognizes a well-defined lipid-storage vesicles class in the NPC patients fibroblasts. A confocal fluorescence microscopy analysis of the cells labelled with sticholysin together with the cholesterol dye filipin and cholera toxin that binds specifically GMI, shows that cholesterol and sphingolipids accumulate in different classes of vesicles. In conclusion, we propose Sticholysin as a novel and valuable tool for the study of the nature and the dynamic of lipid storage in so called lysosomal-diseases.
\end{abstract}

\section{Introduction}

Sphingolipids are ubiquitous and critical components of eukaryotic cell biological membranes typically interacting with cholesterol to drive the formation of plasma membrane rafts [1]. Although previously considered to be simply a structural molecule, sphingomyelin (SM) has an important impact in cell-signalling through its catabolic inter-mediators, such as ceramide and sphingosine, all of these playing crucial roles in the regulation of biological processes [2-4]. Defects in the regulation and metabolism of SM are currently related with Niemann-Pick disease type C (NPC) although the mechanism remains to be clarified [5-7]. NPC is an autosomal recessive syndrome characterized by progressive hepatosplenomegaly and central nervous system neurodegeneration [8]. Approximately $95 \%$ of NPC patients have mutations in NPC1 gene (MIM 607623; chr 18q11- q12), which encodes a glycoprotein of 1,278 amino acids containing 13 transmembrane domains [8]. NPC1 is an integral membrane protein on the limiting membrane of late endosome/lysosome ( $\mathrm{LE} / \mathrm{LY})$ that mediates cholesterol transport from LE/LY to endoplasmic reticulum (ER) and plasma membrane in a vesicle - or oxysterol-binding protein (OSBP) - related protein 5 (ORP5) - dependent manner [9]. Mutations in the NPC1 gene cause a defective mechanism of lipid fate [10] or of the authophagic process [11] with a massive accumulation of intracellular stored granule lipids. The lipid deposit is always composed of non-esterified cholesterol while the presence of different sphingolipides classes is related to the cell type [7]. For example gangliosides and similar glycolipids are present in neurons but not in fibroblast deposits where conversely sphingomyelin is present $[12,13]$. In recent years, the NPC disease
Journal of Toxins
Gabriele Chieppa ${ }^{1 \#}$, Anna Zaratti" ${ }^{1 \#}$, Roberta Taurisano², Federica Deodato², Rosalba Carrozzo $^{2}$,Fiorella Piemonte ${ }^{2}$, Giovanna D'Arcangelo ${ }^{3}$, Claudio Frank ${ }^{4}$, Carlo Dionisi Vici ${ }^{2}$ and Stefano Rufini ${ }^{1^{\star}}$

${ }^{\prime}$ Department of Biology, University of Rome Tor Vergat, 00133 Rome, Italy

${ }^{2}$ Division of Metabolism, Bambino Gesù Children's Hospital, IRCCS, Piazza S. Onofrio 4, 00165 Rome, Italy

${ }^{3}$ Department of Neuroscience, University of Rome Tor Vergat, 00133 Rome, Italy

${ }^{4}$ NationalCentre for Rare Diseases, Istituto Superiore di Sanita', viale Regina Elena, 00161, Rome, Italy

${ }^{\#}$ These authors contributed equally to this work.

*Address for Correspondence

Stefano Rufini, Department of Biology, University of Rome Tor Vergat, 00133 Rome, Italy, Tel: +39.0672594371; Fax: +39062023500; E-mail: rufini@uniroma2.it

Submission: 03 October 2015

Accepted: 23 January, 2016

Published: 27 January, 2016

Copyright: () 2016 Chieppa G, et al. This is an open access article distributed under the Creative Commons Attribution License, which permits unrestricted use, distribution, and reproduction in any medium, provided the original work is properly cited.

Reviewed \& Approved by: Dr. Sung O. Kim, Department of Microbiology and Immunology, Western University, Canada

has become the paradigm of all so-called lisosomal diseases i.e. the pathologies characterized a massive intracellular lipid accumulation. A complete analysis of the nature of intracellular lipid aggregates in lysosomal-disease cells, however, is hindered by the lack of adequate instruments of investigation for lipid recognition. Promising tools to identify lipids are represented by natural products extracted from venom of animals or from bacterial cells able to bind specific lipids. In this study, we compared the cytoplasmic non-esterified cholesterol localization with the sphingomyelin (SM) and GM1 deposits, in both healthy and NPC disease patient fibroblast cultures using the probes Filipin, Cholera toxin (CtxB) and Sticholysin II (Stn II). StnII is a protein extracted from the Stichodactyla helianthus, a sea anemone occurring in the coastal waters of the Caribbean region [14] that shows a cytotoxic activity by forming oligomeric aqueous pores in the cell plasma membrane [15]. StnII belongs to a wider family of protein such as Equinotoxin and Lysenin that bind selectively SM [16] and using a monoclonal antibody anti-StnII (A10) we demonstrated that Stn II is an efficient tool to localise the SM in the plasma membrane of cultured cells [17].

\section{Experimental Procedure}

\section{Patient and controls}

We analysed cultured fibroblasts obtained from skin biopsy of an Italian patient affected by early infantile NPC disease diagnosed and followed Bambino Gesù Children's hospital. He had a history of prenatal splenomegaly, neonatal hepatosplenomegaly, thrombocytopenia and jaundice. At age 18 months developmental delay was also noticed diagnoses was suspected at the age of 2 years 
and 10 months based on bone marrow smear (showing sea-blue histiocytes) and confirmed by genetic analysis of NPC1 (genotype $[c .2339 \mathrm{~T}>\mathrm{G}(\mathrm{p} . \mathrm{V} 780 \mathrm{G})]+[\mathrm{c} .2762 \mathrm{~A}>\mathrm{C}(\mathrm{p} . \mathrm{Q} 921 \mathrm{P})])$ (clinical details of patient have been already reported [18]. Human dermal fibroblasts were obtained from a skin biopsy, after receiving approved informed consent, and grown in DMEM-High glucose medium $(4.5 \mathrm{~g} / \mathrm{L})$ supplemented with $10 \%$ fetal bovine serum and $1 \%$ penicillin and streptomycin. We also used skin fibroblast cells from age-matched controls, that is, children who underwent punch skin biopsy for dermatological diseases.

\section{Chemicals and antibodies}

Tissue culture medium, foetal bovin serum (FBS) and sterile materials were supplied by Flow Laboratories (Irvine, UK); Alexa Fluor 488 cholera toxin subunit B (Fluo-ChTx) conjugate and Alexa Fluor 350 phalloidin, Dextran, BODIPY* FL, 10,000 MW (Bodipydextran) were obtained by Invitrogen (Carlsbad, CA). Filipin and DAPI and all other drugs, media and supplements were obtained from Sigma Chemical Co. Sticholysin II was purified from Stichodactyla heliantus as described by Lanio et al. [15]. Antibodies: 1) Mouse anti-StnII A10 antibody was prepared in our laboratory as described previously [17]. After precipitation with ammonium sulphate from hybridoma culture medium, A10 was dissolved in $\mathrm{Ca}^{2+}$ and $\mathrm{Mg}^{2+}$ free PBS ( $\mathrm{pH}$ 7.4), dialyzed against PBS, and stored frozen as a stock solution. 2) Goat anti-mouse IgG rhodamine conjugated was from (Copper Biomedical) 3) Goat anti-rabbit fluorescein conjugated were from Sigma Chemical Co.

\section{Cell culture}

Cells were grown at $37{ }^{\circ} \mathrm{C}$ in RPMI medium, supplemented with $10 \%(\mathrm{v} / \mathrm{v})$ heat-inactivated FBS, $2 \mathrm{mM}$ glutamine, 0.05 I.U./ml penicillin and 0.05 I.U./ml streptomycin in a humidified atmosphere of $5 \% \mathrm{CO}_{2}$. Fibroblasts from skin biopsies of control individuals (H$\mathrm{DF})$ and patients (NPC-DF) were grown in RPMI 1640 medium containing $10 \%(\mathrm{v} / \mathrm{v}) \mathrm{FBS}$ at $37^{\circ} \mathrm{C}$ in a $5 \% \mathrm{CO}_{2}, 95 \%$ air incubator.

\section{Immunofluorescence}

Cells were plated on $12-\mathrm{mm}$ glass cover-slips at a density of 5 $\mathrm{X} 10^{3} \mathrm{cells} / \mathrm{cm}^{2}$, inserted in $16-\mathrm{mm}$ wells and grown in the RPMI + FBS10\% and then treated with different probes and/or directly fixed in the same well. After 24-48h fibroblasts were treated:

StnII labelled: Cells have been washed with PBS and fixed with $4 \%(\mathrm{w} / \mathrm{v})$ paraformaldehyde (PFA) and after permeabilization with Triton $\mathrm{x}-100,0.1 \%$ for 10 min have been blocked with $1 \%$ bovin serum albumin (BSA-PBS) for $30 \mathrm{~min}$. The cells were then incubated with StnII $\left(4 \mu \mathrm{g} / \mathrm{ml}\right.$ in $1 \%$ BSA-PBS) for $1 \mathrm{~h}$ at $4{ }^{\circ} \mathrm{C}$, washed with PBS and incubated with $\mathrm{A} 10 \mathrm{mAb}$ (1:100 in 10\% BSA solution) for $24 \mathrm{~h}$ at $4{ }^{\circ} \mathrm{C}$, and after extensive rinse in PBS, cells were incubated with a red Texas conjugated goat anti-mouse antibodies diluted in 1\% BSA solution 1:1000 per $1 \mathrm{~h}$, at RT. In some cases the cells were incubated with DAPI (1/1000 in PBS) to the nuclei staining. The slides were mounted using Prolong Antifade kit obtained by Molecular Probes (Eugene, OR).

Filipin - StnII double labelled: Fibroblasts were washed with PBS and fixed with 4\% PFA (w/v) in PBS, washed, and permeabilized with Triton $\mathrm{x}-100 \mathrm{0.1} \%$ for $10 \mathrm{~min}$, blocked with $1 \%$ BSA-PBS for 30 min and stained with StnII as described above, then they are treated with filipin $12.5 \mathrm{mg} / \mathrm{ml}$ for $10 \mathrm{~min}$ RT.

Phalloidin - StnII double labelled: After washing with PBS and fixed with $4 \%(\mathrm{w} / \mathrm{v})$ formaldehyde in PBS, washed, and permeabilized with Triton $\mathrm{x}-100,0.1 \%$ for $10 \mathrm{~min}$, blocked with $1 \%$ BSA-PBS for 30 min and stained with phallodin for $20 \mathrm{~min}$ at room temperature. The cells were then treated with Stn-II as described above.

Cholera Toxin B - StnII double labeleld: The labelling of intracellular GM1 has been carried out as described by Uchida et al. [19]. Fibroblasts were routinely preincubated in serum-free media for $30 \mathrm{~min}$ at $37^{\circ} \mathrm{C}$ before $\mathrm{ChTx}$ uptake. Cells were then pulsed with 10 nM Alexa 488-ChTx and then chased in the medium without ChTx. After washing with PBS and fixed with $4 \%(w / v)$ formaldehyde in PBS, the cells were treated with Stn-II as described above.

Endocytosis was evaluated by incubating living cells for $1 \mathrm{~h}$ at 37 ${ }^{\circ} \mathrm{C}$ with $0.1 \mathrm{mg} / \mathrm{ml}$ of bodipy-dextran. To label the late endosomal vesicles, fibroblasts were incubated as described for an hour and then growth for successive $23 \mathrm{~h}$ in a medium without bodipy-dextran. Cells washed with PBS have been fixed with $4 \%$ (w/v) formaldehyde in PBS, permeabilized with Triton X-100, 0.1\% for $10 \mathrm{~min}$, and finally blocked with $1 \%$ BSA-PBS for $30 \mathrm{~min}$. The cells were then labelled with Stn-II as described above.

\section{Microscopy}

The fluorescence observations were carried out using a Leitz microscope Diaplan (Leica) and images were acquired using a DC250 camera, equipped with a Leica software Q-Fluo, or with a confocal laser scanner microscope (Olympus Fluoview 1000), exciting at 488 $\mathrm{nm}$ with an Ar laser and at $543 \mathrm{~nm}$ with an He laser. To quantify colocalization, the Pearson correlation coefficient ( $r$ ) was determined using FV10-ASW 1,7 Olympus, where ch1i and ch2i are the red and green intensities of voxel $i$, respectively, and chlaver and ch2aver the average value of chli and ch2i, respectively. There is a positive correlation when $\mathrm{r}$ is higher than 0.4 , whereas $\mathrm{r}$ between - 0.4 and 0.4 indicates no correlation [20]. Only for the analysis of the merging of StnII and filipin in the intracellular deposits we use a three-dimensional reconstruction of fluorescent-labelled NPC-DF fibroblasts that were realized by "Surface in 3D rendering" packing of Imaris 6.2 software (Bitplane, Switzerland). In practice, the images of 25 double stained NPC-DF fibroblasts have been taken by confocal microscope and elaborated with imaris program confocal microscope. A total of 530 granules present in random squares (5 $\mu \mathrm{m}^{2}$ ) of the sample fibroblasts have been analyzed to determine the number of filipin-, StnII-, filipin- plus StnII-, positive points.

\section{Results}

Human fibroblasts, obtained from dermal biopsy of healthy donors (H-DF) and NPC1 patients (NPC-DF), were treated with StnII and subsequently with A10 Antibodies. Images obtained by fluorescence microscopy show that in H-DF only a diffuse red staining is detectable, this is associated to the plasma membranes (Figure 1a) while in NPC-DF, StnII dyes brightly discrete granules (Figure 1b). A more accurate localization of StnII-positive granules inside the NPC-DF cell was obtained by cell analysis after observation with confocal microscopy (Figures 1c-1g). The granules in the cytoplasm 
Citation: Chieppa G, Zaratti A, Taurisano R, Deodato F, Carrozzo R. Sticholysin II Identifies Intracellular Lipid Deposits in Niemann Pick C Fibroblasts. J Toxins. 2016;3(1): 3
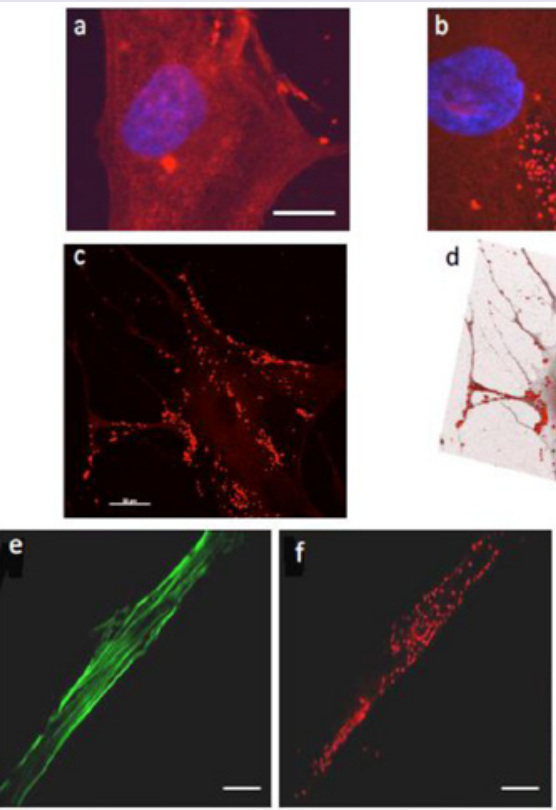

Figure 1: Stnll localization in H-DF and NPC-DF fibroblasts.

H-DF (a) and NPC-DF (b) were incubated with Stnll and treated with A10-anti-Stn-II and antimouse rhodamine and counterstained with DAPI and observed at $100 x$ magnification. Granule associated Stnll has been observed in NPC-DF by confocal microscopy (c) and 3D images reconstructed by processing software (d). Fibroblasts were simultaneously labelled with phalloidin (e) and Stnll (f) and the images were merged (g). Scale bars correspond to $5 \mu \mathrm{m}$ (a-b) $30 \mu \mathrm{m}$ (c-f) $15 \mu \mathrm{m}$ (g)

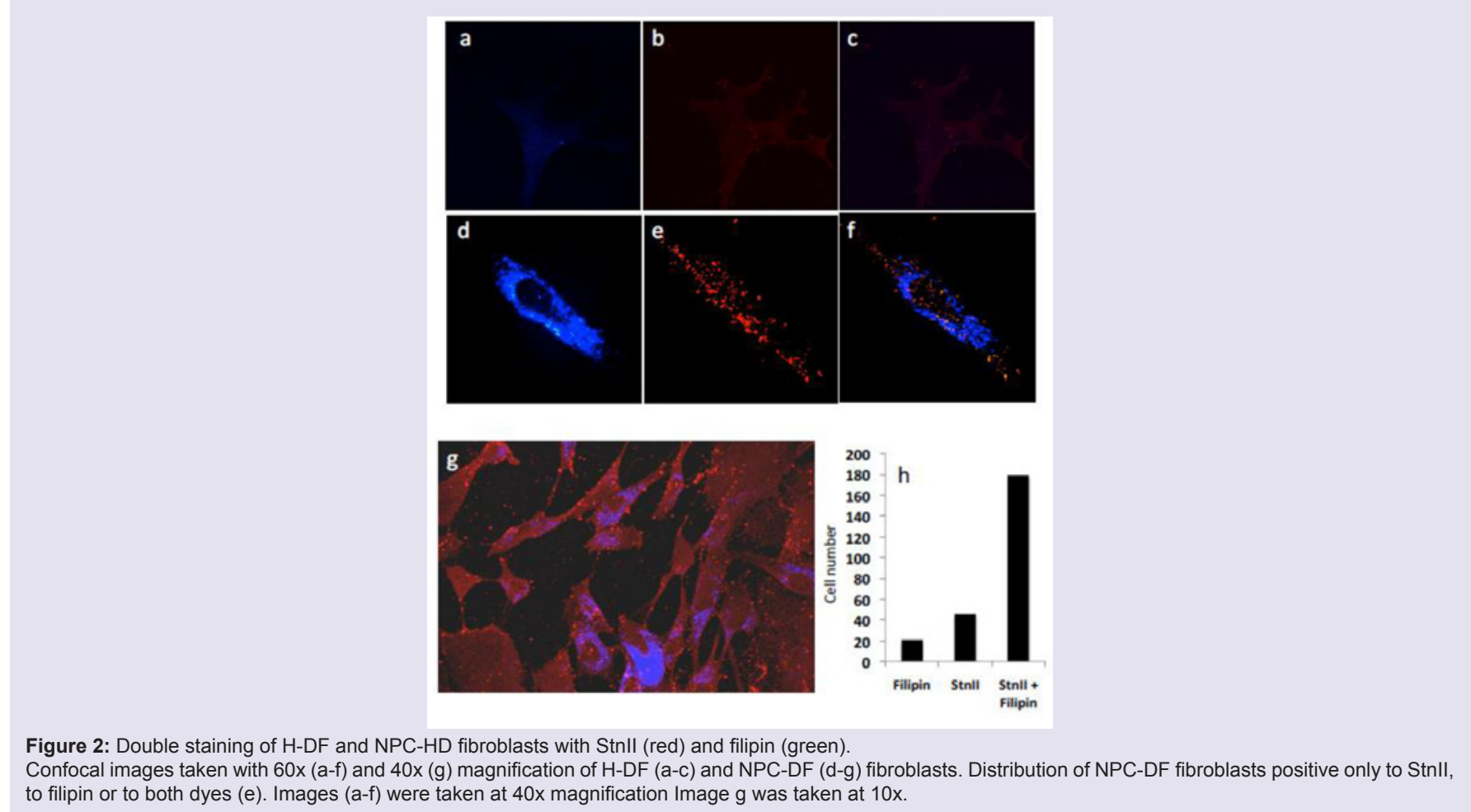

have a diameter between 100 and $300 \mathrm{~nm}$ and they show a random distribution (Figures $1 \mathrm{c}-1 \mathrm{~d}$ ) without any apparent association with either plasma membrane or nucleus. To study the connection between the microfilaments network and lipid granules we double stained NPC-DF cells with StnII (red) together the florescent F-actin probe, phalloidin (green). The images obtained by confocal microscopy show the presence of well-defined stress fibbers (Figure 1e) as well StnII-labelled granules (Figure 1f). The probes do not show a colocalization suggesting that the granules are not associated with subcortical actin filaments (Figure 1g). $29 \mathrm{H}-\mathrm{DF}$ and NPC-DF fibroblasts 
Citation: Chieppa G, Zaratti A, Taurisano R, Deodato F, Carrozzo R. Sticholysin II Identifies Intracellular Lipid Deposits in Niemann Pick C Fibroblasts. J Toxins. 2016;3(1): 3
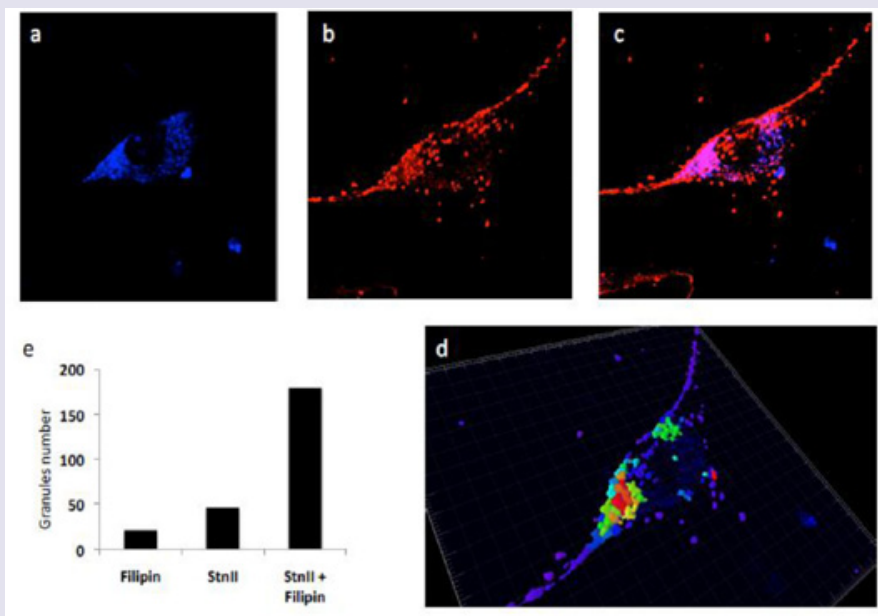

Figure 3: Analysis of intracellular localization of Filipin and Stnll in NPC-DF fibroblasts.

Representative images of NPC-DF fibroblast double stained with Filipin (blue - a), Stnll (red - b) and their co-localization (c). Three dimensional merging of the probes localized in internal stores after 3D images analysis by software Imaris 6.2 , indicating the presence of three different stores pools (d). Distribution of the intracellular store pools (e): i.e. positive only to Stnll or filipin and to both dyes. Images were taken at 40x magnification.

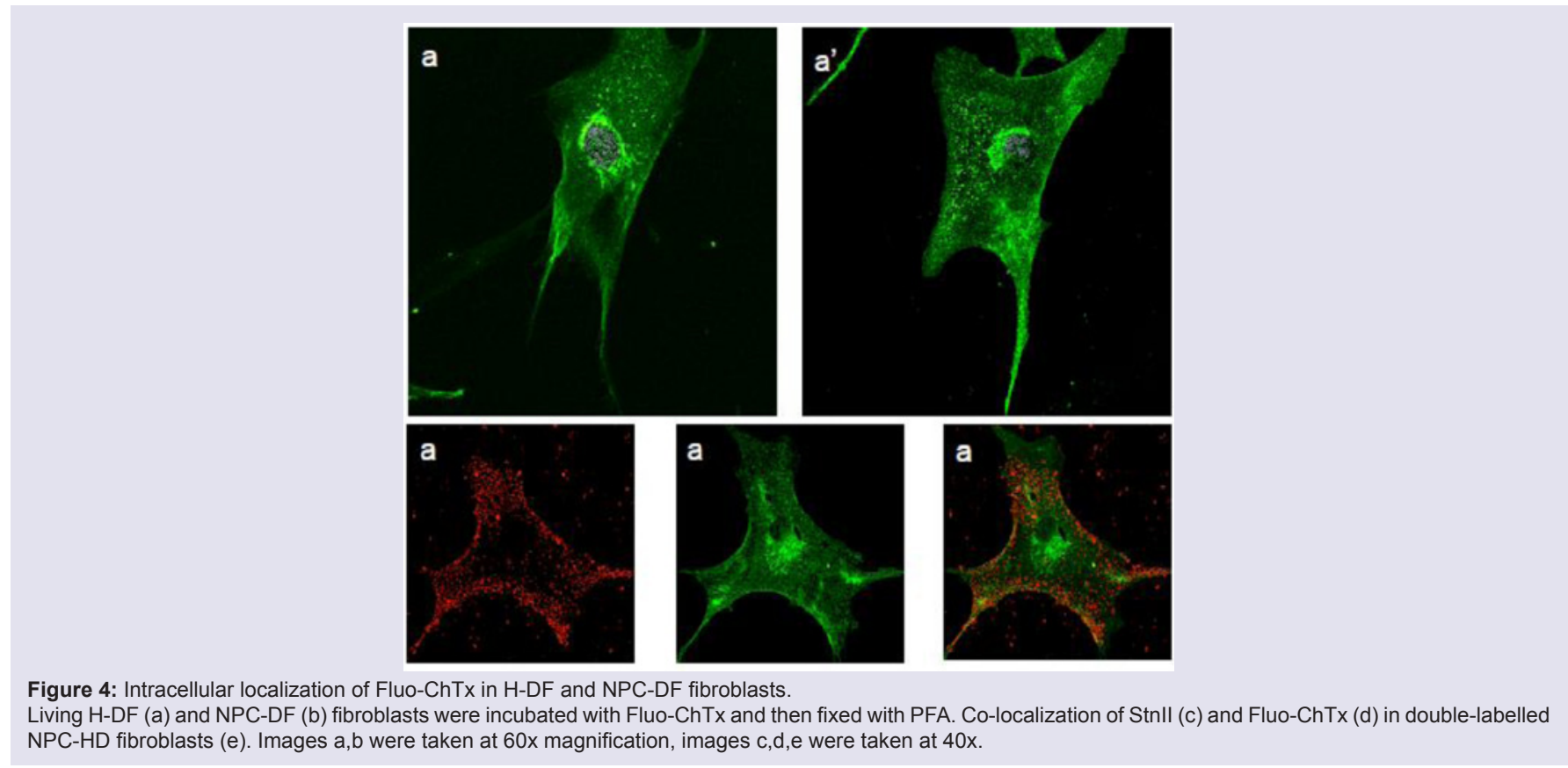

were double stained with StnII (red) and with filipin, a cholesterol fluorescent probe (blue) (Figure 2). Filipin (blue) and StnII (red) reveal the presence of intracellular cholesterol deposit and SM respectively in NPC-HD cells (Figures 2d-2f) but not in the control fibroblasts which appear stained only faintly and without any lipid deposit in the cytoplasm (Figures 2a-2c). Although most of the NPC-DF fibroblasts is stained at once by the toxin and filipin, some cells are positive only for one of the two dyes (Figure 2f). The microscopic analysis of 250 cells from five different slides, has revealed that the majority of cells (75\%) was positive to both probes, while the $16 \%$ only to StnII and the remaining $9 \%$ only to filipin (Figure $2 \mathrm{~g}$ ). To gain more information on the lipid composition of intracellular granules, StnII and filipin double-stained NPCDFs were analysed by confocal microscope (Figure 3); pictures obtained were elaborate with a "Surface in 3D rendering" and were analysed to evaluate the co-presence of the two dyes in vesicular structures (Figure $3 \mathrm{~d}$ ) of a sample of 25 fibroblasts. The analysis of the merging of fluorescent dyes reveals that fewer than $30 \%$ of the granules containing filipin also contain a quantifiable presence of StnII, suggesting the presence in NPC-DF of at least three different granule pools (Figure 3e). H-DF and NPC-DF fibroblasts were stained with Fluo-ChTx, a GM1 fluorescent probe (Figure 4) using the method of Uchida et al. in which, after the binding of the probe to the membrane, the subsequent incubation allows the complete endocytosis of the ChTx-GM1 complex [19]. This method permits to study the intracellular localization of the GM1 as show in Figure 4. Fluo-ChTx, faintly stained the plasma membrane while it strongly accumulates in the vesicles around the nucleus, which could tentatively refer to the Golgi apparatus, in both H-DF (Figure 4a) and 

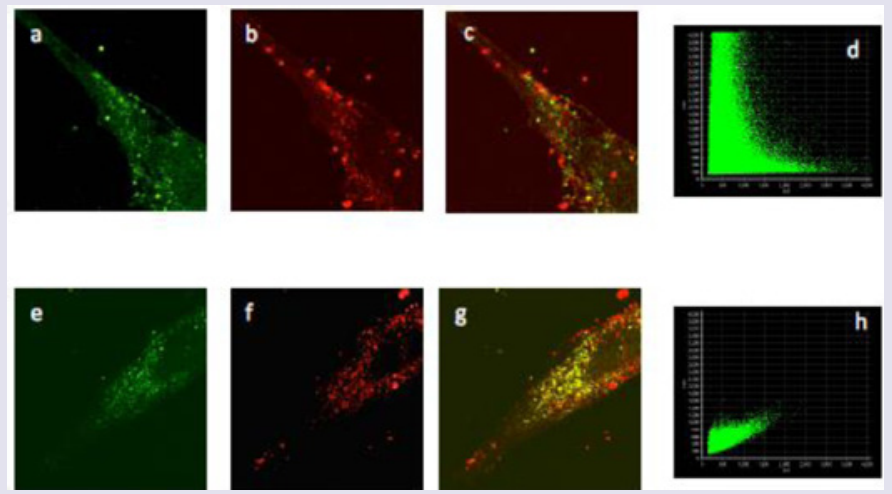

Figure 5: Intracellular localization of dextran and Stnll in NPC-DF.

Representative images of NPCDF fibroblast double stained with bodipy-dextran (green - a,e), Stnll (red - b,f) and their colocalization (c,g). Living fibroblasts were labelled for 1 (a-d) or 24 hours (e-h) with bodipydextran and, after fixation and permeabilization, with Stn-II (red). Index of co-localization in cell treated for 1 (d) or $24 \mathrm{~h}$ with bodipy-dextran of channels: Ch1 (dextran) and Ch2 (Stn-II). Images were taken at 100x magnification.

NPC-DF fibroblasts (Figure 4b). We did not appreciate any evident differences of the Fluo-ChTx fluorescence intensity between the H-DF and NPC-DF cells suggesting a similar amount of total GM1. The analysis of StnII (Figure 4c) and Fluo-ChTx (Figure 4d) double staining NPC-DF fibroblasts do not indicate a co-localization of the two probes in the intracellular granules (Figure 4e), suggesting that GM1 is not present in the same SM accumulation compartments. The presence of SM accumulation in the early and late endosomal compartments has been analyzed by labeling the cells with StnII after the endocytosis by NPC-DF of the fluorescent polysaccharide dextran (Figure 5). After $1 \mathrm{~h}$ of incubation, a large amount of bodipy-dextran accumulates into intracellular vesicles (Figure 5a) which are referable to early-endosomes [21]. In this condition, StnII does not show any colocalization with bodipy-dextran (Figures $5 \mathrm{c}$ and $5 \mathrm{~d}$ ) indicating that SM deposits are not localized in the early-endosome compartment. To stain the late-endosome vesicles, NPC-DF fibroblasts were growth $1 \mathrm{~h}$ in presence of bodipy-dextran and then in a medium free of the fluorescent for subsequent $23 \mathrm{~h}$ and finally treated with StnII (Figures $5 e-5 h)$. Images indicate a partial co-localization of StnII with bodipydextran, as demonstrated by a value of the coefficient of Pearson greater than 0.6. This data suggest that, in NPC-DF fibroblasts, the late endosomes are accumulation sites of SM.

\section{Discussion}

In all the lysosomal-pathology is observed an intra-cellular lipid accumulation in different tissues, although there are very few data about their exact role in the disease progression [22]. In the NPC patient's cells, the lipids that accumulate are mainly cholesterol and SM in peripheral tissues and neutral glycosphingolipid in brain tissue with only little excessive cholesterol level [23,24]. In this paper we study the ability of Sticholysin-II to detect the presence of SM in the intracellular lipid deposits of NPC fibroblasts and we correlate the SM deposits with GM1 and cholesterol ones, using other fluorescent probes: i.e. ChTx for GM1 ganglioside [25] and filipin for cholesterol [26]. In respect to the control cells, the StnII stains clearly recognizable cytoplasmic granules in the NPCDF, suggesting an intracellular vesicular deposit of SM in the cytoplasm. A cholesterol but not a sphingolipids accumulation in late-endosomal compartments in the NPC fibroblasts has been described by different authors [13]. The only available data concerning SM intracellular accumulation in sphingolipidosis patients' cells do indeed refer to Niemann-Pick A (NPA) peripheral cell cultures. These data have been obtained using two different strategies: by cell incubation with analogues fluorescent of ceramide or by the use of GFP-conjugated lysenin [27-29].

Although several diagnostic, pathological and cytological aspects link NPA to NPC disease, there is a difference regarding the nature of the intracellular SM accumulation. In NPA it results from the inhibition of acidic-sphingomyelinase derived by the direct gene mutation whereas it has been proposed that in NPC cells, the sphingolipid accumulation resulting from a secondary effect induced by high cholesterol concentration [30,31]. In NPC fibroblasts, the SM is associated with cholesterol only in the $25-30 \%$ of vesicles as showed by the co-localization of filipin with StnII. The presence of at least three different vesicles (i.e. containing SM or cholesterol alone and $\mathrm{SM} /$ Cholesterol together) implies that the intra-cellular traffic of these lipids is not always associated, also if the NPC protein is involved in their correct maturation. A possible hypothesis would explain our results could involve the different endo-lysosomal maturation and the degree of the cholesterol accumulation. Accordingly, double stained vesicles correspond to endolysosomal compartment with a concentration of cholesterol sufficiently high to inhibit SMase inducing a secondary accumulation of SM as demonstrated by Blom et al. [31]. Alternatively the double stained vesicles could represent vesicles of neo-synthesized lipid (both SM and cholesterol) and not derived from internalized lipoproteins, in agreement with Zhang's study which suggests the exclusive presence of NPC1 protein on the vesicles with cholesterol synthesised by cells and not by the LDL proteins or by recycling [26]. We cannot exclude skewed results, depending on the possible property of StnII to recognize only one "structural arrangement" of SM, which is present mainly in the cholesterol-free granules. Evidence indicates that different SM-binding proteins i.e. Lysenine (Lys) from coelomic of Eisenia foetida and Equinotoxin-II (EqII) from Actinia equina, can recognize SM only in a particular structural confirmation: Lys prefers SM in clustered conformation and EqII in a more disordered phase [14]. Yachi et al. treating cells with both proteins identified two classes of SM-enriched vesicles, one referred as late endosome and other one as recycling endosome in line with the different localization of the two 
Citation: Chieppa G, Zaratti A, Taurisano R, Deodato F, Carrozzo R. Sticholysin II Identifies Intracellular Lipid Deposits in Niemann Pick C Fibroblasts. J Toxins. 2016;3(1): 3

ISSN: 2328-1723

main isoforms of sphingomyelin synthase, SMS2 and SMS1, located in plasma- and endo- membranes respectively [14]. Our images show only a negligible difference in the total fluorescence between H-DF and NPC-DF, a result that matches previously published observations on the lack of glycolipid deposits in peripheral tissue [32]. In our own experimental conditions, ChTx stained preferentially the internalized GM1 [19] as demonstrated by the presence of fluorescence mainly in the peri-nuclear vesicular structure, referred to endoplasmic reticulum/Golgi apparatus and in very few internal vesicles. The peri-nuclear structures of NPC-DF in respect to the H-DF did not show any increase of GM1 associated fluorescence whereas little but constant differences are present in the cytoplasm vesicles. Sugimoto et al. reports that in $\mathrm{CHO}$ cells, after internalization, GM1 take at least two pathways: the way back to the plasma membrane and the way to the endoplasmic reticulum via the Golgi apparatus, and that only this way depends on the NPC1's function, whereas transport to the Golgi apparatus/ER does not [33]. Taking into account this hypothesis, the increase in the fluorescence of the cytoplasm vesicles in the NPC-DF cells are probably related to the impairment of the recycling of the GM1/ChTx complex after internalization. Finally, we studied the localization of the toxin in the early and late endosome compartment, using the different times of incorporation of a polysaccharide with high molecular weight. The results show that SM accumulates preferentially in the late-endosome compartment, as previously observed using different experimental approaches $[13,21]$.

We conclude that StnII/A10 gave exciting finding about the localization and the nature of the different lipid deposits in NPCderived cells that could improve the knowledge of lysosomal diseases.

\section{References}

1. Chiantia S, London E (2013) Sphingolipids and membrane domains: recent advances. Handb Exp Pharmacol 215: 33-55.

2. Taniguchi M, Okazaki T (2014) The role of sphingomyelin and sphingomyelin synthases in cell death, proliferation and migration-from cell and animal models to human disorders. Biochim Biophys Acta 1841: 692-703.

3. García-Barros M, Coant N, Truman JP, Snider AJ, Hannun YA (2014) Sphingolipids in colon cancer. Biochim Biophys Acta 1841: 773-782.

4. van Echten-Deckert G, Walter J (2012) Sphingolipids: critical players in Alzheimer's disease. Prog Lipid Res 51: 378-393.

5. Vance JE (2006) Lipid imbalance in the neurological disorder, Niemann-Pick C disease. FEBS Lett 580: 5518-5524.

6. Schulze H, Sandhoff K (2014) Sphingolipids and lysosomal pathologies. Biochim Biophys Acta 1841: 799-810.

7. Fan M, Sidhu R, Fujiwara H, Tortelli B, Zhang J, et al. (2013) Identification of Niemann-Pick $\mathrm{C} 1$ disease biomarkers through sphingolipid profiling. J Lipid Res 54: 2800-2814.

8. Vanier MT, Millat G (2003) Niemann-Pick disease type C. Clin Genet 64: 269-281.

9. Yu XH, Jiang N, Yao PB, Zheng XL, Cayabyab FS, et al. (2014) NPC1, intracellular cholesterol trafficking and atherosclerosis. Clin Chim Acta. 429: 69-75

10. Lloyd-Evans E, Morgan AJ, He X, Smith DA, Elliot-Smith E, et al. (2008) Niemann-Pick disease type $\mathrm{C} 1$ is a sphingosine storage disease that causes deregulation of lysosomal calcium. Nat Med 14: 1247-1255.

11. Pacheco CD, Elrick MJ, Lieberman AP (2009) Tau normal function influences Niemann- Pick type $\mathrm{C}$ disease pathogenesis in mice and modulates autophagy in NPC1-deficient cells. Autophagy 5: 548-550.
12. Zervas M, Dobrenis, K, Walkley SU (2001) Neurons in Niemann-Pick disease type $C$ accumulate gangliosides as well as unesterified cholesterol and undergo dendritic and axonal alterations. J Neuropathol Exp Neurol 60: 4964

13. Sztolsztener ME, Dobrzyn A, Pikulas S, Tylki-Szymanska A, BandorwiczPikula J (2012) Impaired dynamics of the late endosome/lysosome compartment in human Niemann-Pick type $\mathrm{C}$ skin fibroblasts carrying mutation in NPC1 gene. Mol Biosyst 8: 1197-1205

14. Yachi R, Uchida Y, Balakrishna BH, Anderluh G, Kobayashi T, et al. (2012) Subcellular localization of sphingomyelin revealed by two toxin-based probes in mammalian cells. Genes Cells 17: 720-727.

15. Ros U, Edwards MA, Epand RF, Lanio ME, Schreier S, et al. (2013) The sticholysin family of pore-forming toxins induces the mixing of lipids in membrane domains. Biochim Biophys Acta 1828: 2757-2762.

16. Lanio ME, Morera V, Alvarez C, Tejuca M, Gómez T, et al. (2001) Purification and characterization of two hemolysins from Stichodactyla helianthus. Toxicon 39: 187-194.

17. Garcia PS, Chieppa G, Desideri A, Cannata S, Romano E, et al. (2012) Sticholysin II: a pore-forming toxin as a probe to recognize sphingomyelin in artificial and cellular membranes. Toxicon 60: 724-733.

18. Ginocchio VM, D’Amico A, Bertini E, Ceravolo F, Dardis A, et al. (2013) Efficacy of miglustat in Niemann-Pick $C$ disease: a single centre experience. Mol Genet Metab 110: 329-335.

19. Uchida Y, Hasegawa J, Chinnapen D, Inoue T, Okazaki S, et al. (2011) Intracellular phosphatidylserine is essential for retrograde membrane traffic through endosomes. Proc Natl Acad Sci U S A 108: 15846-15851.

20. Costes SV, Daelemans D, Cho EH, Dobbin Z, Pavlakis G, et al. (2004) Automatic and quantitative measurement of protein-protein colocalization in live cells. Biophys J 86: 3993-4003.

21. Rappaport J, Manthe RL, Solomon M, Garnacho C, Muro S (2016) A Comparative study on the alterations of endocytic pathways in multiple lysosomal storage disorders. Mol Pharm [Epub ahead of print].

22. Frolov A, Srivastava K, Daphna-Iken D, Traub LM, Schaffer JE, et al. (2001) Cholesterol overload promotes morphogenesis of a Niemann-Pick C (NPC)like compartment independent of inhibition of NPC1 or HE1/NPC2 function. J Biol Chem 276: 46414-46421.

23. Vanier MT (1999) Lipid changes in Niemann-Pick disease type C brain personal experience and review of the literature. Neurochem Res 24: $481-$ 489.

24. Zhang M, Dwyer NK, Love DC, Cooney A, Comly M, et al. (2001) Cessation of rapid late endosomal tubulovesicular trafficking in Niemann-Pick type C1 disease. Proc Natl Acad Sci U S A 98: 4466-4471.

25. Fuxe K, Tinner B, Janson AM, Cintra A, Staines W, et al. (1989) On the cellular localization and distribution of the ganglioside GM1 in the rat brain as revealed by immunofluorescence histochemistry of cholera toxin binding sites. Acta Physiol Scand 137: 551-552.

26. Gimpl G, Gehrig-Burger K (2007) Cholesterol reporter molecules. Biosci Rep 27: $335-358$

27. Koval M, Pagano RE (1989) Lipid recycling between the plasma membrane and intracellular compartments: transport and metabolism of fluorescent sphingomyelin analogues in cultured fibroblasts. J Cell Biol 108: 2169-2181.

28. Yamaji A, Sekizawa Y, Emoto K, Sakuraba H, Inoue K, et al. (1998) Lysenin, a novel sphingomyelin-specific binding protein. J Biol Chem 273: 5300-5306.

29. Taksir TV, Johnson J, Maloney CL, Yandl E, Griffiths D, et al. (2012) Optimization of a histopathological biomarker for sphingomyelin accumulation in acid sphingomyelinase deficiency. J Histochem Cytochem 60: 620-629.

30. Devlin C, Pipalia NH, Liao X, Schuchman EH, Maxfield FR, et al. (2010) Improvement in lipid and protein trafficking in Niemann-Pick $\mathrm{C} 1$ cells by correction of a secondary enzyme defect. Traffic 11: 601-615. 
Citation: Chieppa G, Zaratti A, Taurisano R, Deodato F, Carrozzo R. Sticholysin II Identifies Intracellular Lipid Deposits in Niemann Pick C Fibroblasts. J Toxins. 2016;3(1): 3

ISSN: $2328-1723$

31. Blom T, Li Z, Bittman R, Somerharju P, Ikonen E (2012) Tracking sphingosine metabolism and transport in sphingolipidoses: NPC1 deficiency as a test case. Traffic 13: 1234-1243.

32. Penchev P, Vanier M, Suzuki K, Patterson M, Scriver CR, et al. (1994) The metabolic and molecular bases of inherited disease, ( $7^{\text {th }}$ edn). New York: McGraw-Hill, pp. 2625-2639.

33. Sugimoto Y, Ninomiya H, Ohsaki Y, Higaki K, Davies JP, et al. (2001) Accumulation of cholera toxin and GM1 ganglioside in the early endosome of Niemann-Pick C1-deficient cells. Proc Natl Acad Sci U S A 98: 12391-12396.

Acknowledgements

We are indebted to Mr. Martin Bennet for revising the manuscript and with Dr. Elena Romano for confocal images. 